\title{
INGESTA ORAL DO PACIENTE HOSPITALIZADO COM DISFAGIA OROFARÍNGEA NEUROGÊNICA
}

\author{
Oral Intake of hospitalized patient \\ with neurogenic oropharyngeal dysphagia
}

Carolina Castelli Silvério ${ }^{(1)}$, Ana Maria Hernandez ${ }^{(2)}$, Maria Inês Rebelo Gonçalves ${ }^{(3)}$

\section{RESUMO}

Objetivo: verificar a evolução na ingesta oral e a ocorrência de broncopneumonias (BCP) em pacientes hospitalizados com disfagia orofaríngea neurogênica, após atuação fonoaudiológica. Métodos: 50 pacientes adultos, divididos em grupos: I: 31 pacientes pós-acidente vascular encefálico; II: sete pacientes pós-traumatismo crânio-encefálico; III: 12 pacientes com demência. Foram levantadas as informações antes e após a atuação fonoaudiológica: nível da Functional Oral Intake Scale (FOIS), ocorrência de BCP; número de atendimentos fonoaudiológicos e motivo de interrupção destes. Resultados: houve aumento significativo dos níveis da escala FOIS e redução do percentual de ocorrência de BCP nos três grupos estudados. Nos grupos pós-AVE e demência a interrupção da fonoterapia ocorreu devido à alta hospitalar, enquanto que no grupo pós-TCE devido à alta fonoaudiológica. Conclusão: os pacientes deste estudo demonstraram avançar das consistências alimentares na ingesta oral, e redução da ocorrência de $\mathrm{BCP}$, após a intervenção fonoaudiológica com relação à disfagia.

DESCRITORES: Transtornos da Deglutição; Fonoterapia; Acidente Cerebral Vascular; Traumatismos Encefálicos; Demência

\section{INTRODUÇÃO}

Pacientes hospitalizados com alterações neurológicas, doenças neurodegenerativas, alterações anatômicas de cabeça e pescoço, e/ou com comprometimentos respiratórios, podem apresentar como sintoma de sua patologia a Disfagia Orofaríngea (DO). A DO compreende uma alteração no processo da deglutição, a qual pode comprometer

(1) Fonoaudióloga da Associação de Assistência à Criança Deficiente, AACD, São Paulo, SP; Mestre em Ciências pela Universidade Federal de São Paulo.

(2) Fonoaudióloga Chefe da Equipe de Fonoaudiologia do Hospital Santa Catarina, São Paulo, SP; Mestre em Distúrbios da Comunicação pela Pontifícia Universidade Católica de São Paulo.

(3) Fonoaudióloga; Professora Adjunta do Departamento de Fonoaudiologia da Universidade Federal de São Paulo, UNIFESP, São Paulo, SP; Chefe do Serviço Integrado de Fonoaudiologia do Hospltal São Paulo - Universidade Federal de São Paulo; Pós-doutorado pela University of California.

Conflito de interesses: inexistente aspectos clínicos, nutricionais e/ou sociais do indivíduo ${ }^{1,2}$.

A disfagia ocorre quando há um descontrole na coordenação das funções de respiração e alimentação e pode ocorrer como resultado de alterações neurológicas congênitas ou adquiridas, estruturais ou funcionais, ou ainda como consequência de estados mórbidos ${ }^{3,4}$. Silva et al ${ }^{5}$. referem que o acidente vascular encefálico (AVE) acomete a neurofisiologia da deglutição, afetando as vias de transmissão córtiço-bulbares. A aspiração pulmonar pode ser fatal e a má nutrição pode ocorrer quando a alteração não é diagnosticada de forma correta, reduzindo a imunidade do paciente, deixando-o mais suscetível à aquisição de doenças ${ }^{6}$. Segundo Broadley et al. ${ }^{7}$, a presença de disfagia é comum após o AVE, e está relacionada com sua morbidade e mortalidade. Alterações na dinâmica da deglutição proporcionam respostas psico-sociais como ansiedade, medo, insegurança e redução da autoestima, em decorrência do aspecto social relacionado com as atividades de comer e beber ${ }^{8}$. 
Diante da DO, o profissional fonoaudiólogo avalia e reabilita o processo de deglutição deste paciente. No atendimento ao paciente disfágico hospitalizado compete ao fonoaudiólogo colaborar juntamente com a equipe interdisciplinar na melhora clínica do paciente, podendo reduzir o tempo de uso das vias alternativas de alimentação, o tempo de hospitalização, e contribuir para a melhora do quadro pulmonar.

Segundo DePippo et al. ${ }^{9}$ o objetivo da reabilitação no atendimento a pacientes com distúrbios da deglutição consiste na estabilização do estado nutricional e na eliminação dos riscos de complicações clínicas decorrentes da aspiração laringotraqueal.

De acordo com Angelis et al. ${ }^{10}$, a fonoaudiologia dificilmente normalizará a função de deglutição, mas a contribuição de seus conhecimentos para maximização deste processo e para o estabelecimento da alimentação por via oral o mais precoce possível, é inegável.

A evolução da ingestão oral segura e funcional do paciente, associada à manutenção da saúde pulmonar e de seu estado nutricional é uma evidência significativa da eficácia terapêutica. Surge assim à necessidade de se verificar com que via de alimentação, e com quais consistências alimentares, quando se tratar de uma alimentação por via oral, o paciente com disfagia orofaríngea apresentava no início e no término da atuação fonoaudiológica.

Assim, o objetivo deste estudo foi verificar a evolução da consistência alimentar ingerida e a ocorrência de broncopneumonias (BCP), em pacientes hospitalizados com disfagia orofaríngea neurogênica sugiro colocar desde o título que é neurogênica, que receberam a atuação fonoaudiológica frente à disfagia.

\section{MÉTODOS}

O estudo foi realizado de forma retrospectiva. Os dados levantados foram obtidos mediante a análise das evoluções da atuação fonoaudiológica no prontuário médico dos pacientes atendidos pela equipe de fonoaudiologia nos anos de 2005 e 2006.

Participaram deste estudo 50 pacientes adultos, que se encontravam internados no Hospital Santa Catarina, tanto em unidades de tratamento intensivo como em leitos hospitalares, que apresentaram como sintomatologia a presença de disfagia orofaríngea neurogênica, e foram submetidos à fonoterapia. Os pacientes foram divididos em três grupos, de acordo com a patologia médica de base, sendo: Grupo I: 31 pacientes pós-AVE, com média de idade de 73 anos; Grupo II: sete pacientes póstraumatismo crânio-encefálico (TCE), com média de idade de 52 anos; Grupo III: 12 pacientes com diagnóstico de demência, apresentando média de idade de 82 anos.

Foram levantadas as seguintes informações no primeiro e no último dia da atuação fonoaudiológica:

- Via de alimentação utilizada e consistências alimentares ingeridas, no caso da alimentação ocorrer por via oral. Os pacientes eram então classificados de acordo com a Escala Funcional de Alimentação (Functional Oral Intake Scale FOIS ${ }^{11}$ ), que compreende uma escala de 7 níveis, que classifica o paciente quanto ao nível de alimentação por via oral, sendo eles: nível l: nada por via oral. Compreende o paciente que se alimenta exclusivamente por via alternativa de alimentação; nível II: dependência de via alternativa, com mínima oferta de via oral. São os pacientes que se alimentam por via alternativa de alimentação, mas recebem estímulos gustativos, ou ofertas ocasionais de pequeno volume por via oral; nível III: dependência de via alternativa, com oferta consistente de via oral. Representam os pacientes que se nutrem por via alternativa, mas também ingerem via oral, geralmente de uma única consistência, mais para prazer alimentar; nível IV: via oral total, mas limitada a uma única consistência. Quando o indivíduo recebe somente alimentação por via oral, sendo esta de somente uma consistência; nível $V$ : via oral total, com mais de uma consistência, mas que necessitam de preparo especial. Compreendem os indivíduos que podem ingerir mais de uma consistência de alimento, mas este precisa ser especialmente preparado, como, por exemplo, ser bem cozido ou batido; nível VI: via oral total, com mais de uma consistência, que não necessitam de preparo especial, mas apresenta limitações específicas do alimento. Quando o paciente por ingerir múltiplas consistências sem preparo especial, mas apresenta algumas restrições, como por exemplo, líquidos somente gaseificados, ou restrição de grãos secos; nível VIl: via oral total, sem restrições.

- Ocorrência de broncopneumonias (BCP) antes e durante a intervenção fonoaudiológica;

- Número de atendimentos fonoaudiológicos e o motivo de interrupção destes, podendo ocorrer por alta do atendimento fonoaudiológico, alta hospitalar, suspensão do atendimento por intercorrências clínicas e óbito. Os atendimentos ocorreram diariamente, com freqüência de 6 vezes na semana, iniciando com a solicitação médica de intervenção fonoaudiológica.

Esta pesquisa foi aprovada pelo Comitê de Ética em Pesquisa do Hospital Santa Catarina CEP № 018/06. 
Para a análise dos resultados foi aplicado o Teste de Kruskal-Wallis, para verificar possíveis diferenças entre os três grupos estudados, quando comparados concomitantemente e o Teste de McNemar, para verificar possíveis diferenças entre os dois momentos de observação. Adotamos o nível de significância de 5\% $(0,005)$.

\section{RESULTADOS}

Com relação à aplicação da escala FOIS (Tabela 1), observou-se que nos três grupos de pacientes, houve aumento de nível na escala FOIS, com diferença estatisticamente significante. A maior parte dos pacientes dos três grupos encontrou-se no Nível I da escala antes da intervenção fonoaudiológica, e após tal intervenção, encontrou-se em sua maioria no nível $V$ nos pacientes pós-AVE e com demência e no nível VII nos pós-TCE.

$\mathrm{Na}$ Tabela 2 foi possível observar os resultados referentes à presença de $\mathrm{BCP}$ antes e após a intervenção fonoaudiológica, com diferenças estatisticamente significantes em todos os grupos estudados, com redução do percentual de ocorrência da mesma.

O motivo da interrupção da intervenção fonoaudiológica encontra-se descrito na Tabela 3 , evidenciando diferença significante entre os motivos levantados, com maior percentual de pacientes com interrupção por alta hospitalar nos pacientes com demência e pós-AVE, e por alta fonoaudiológica naqueles pós-TCE. A média do número de atendimentos (Tabela 4) dos três grupos estudados não apresentou diferença estatisticamente significante entre eles, com maior média o grupo pós-TCE.

Tabela 1 - Distribuição numérica e percentual dos grupos de pacientes em relação à Escala FOIS, pré e pós-intervenção fonoaudiológica

\begin{tabular}{|c|c|c|c|c|c|c|}
\hline \multirow{4}{*}{ Nível FOIS } & \multicolumn{6}{|c|}{ Patologia } \\
\hline & \multicolumn{2}{|c|}{$\operatorname{AVE}\left(p<0,001^{*}\right)$} & \multicolumn{2}{|c|}{ TCE $\left(p=0,023^{\star}\right)$} & \multicolumn{2}{|c|}{ Demência $(p=0,007)$} \\
\hline & pré & pós & pré & pós & pré & pós \\
\hline & $N(\%)$ & $\mathbf{N}(\%)$ & N (\%) & N (\%) & N (\%) & N (\%) \\
\hline 1 & $28(90,32)$ & $5(16,13)$ & $7(100)$ & $1(14,29)$ & $10(83,33)$ & $2(16,67)$ \\
\hline II & $0(0)$ & $0(0)$ & $0(0)$ & $0(0)$ & $0(0)$ & $2(16,67)$ \\
\hline III & $0(0)$ & $1(3,23)$ & $0(0)$ & $0(0)$ & $0(0)$ & $1(8,33)$ \\
\hline IV & $2(6,45)$ & $3(9,68)$ & $0(0)$ & $0(0)$ & $1(8,33)$ & $2(16,67)$ \\
\hline V & $1(3,23)$ & $17(54,84)$ & $0(0)$ & $2(28,57)$ & $1(8,33)$ & $4(33,33)$ \\
\hline $\mathrm{VI}$ & $0(0)$ & $4(12,90)$ & $0(0)$ & $0(0)$ & $0(0)$ & $1(8,33)$ \\
\hline VII & $0(0)$ & $1(3,23)$ & $0(0)$ & $4(57,14)$ & $0(0)$ & $0(0)$ \\
\hline Total & $31(100)$ & $31(100)$ & $7(100)$ & 7 (100) & $12(100)$ & $12(100)$ \\
\hline
\end{tabular}

Legenda: FOIS: Functional Oral Intake Scale; AVE: acidente vascular encefálico; TCE: traumatismo crânio-encefálico; N: número de pacientes; $p=$ significância

Tabela 2 - Distribuição numérica e percentual dos grupos de pacientes em relação à presença de broncopneumonias (BCP) antes e após intervenção fonoaudiológica

\begin{tabular}{|c|c|c|c|}
\hline \multirow{3}{*}{ BCP } & \multicolumn{3}{|c|}{ Patologia } \\
\hline & $\operatorname{AVE}\left(p<0,001^{*}\right)$ & TCE $\left(p=0,046^{*}\right)$ & Demência $\left(p=0,002^{*}\right)$ \\
\hline & $N(\%)$ & $N(\%)$ & $\mathrm{N}(\%)$ \\
\hline Pré & $25(80,65)$ & $4(57,14)$ & $11(91,67)$ \\
\hline Pós & $2(6,45)$ & $0(0)$ & $1(8,33)$ \\
\hline
\end{tabular}

Legenda: FOIS: Functional Oral Intake Scale; AVE: acidente vascular encefálico; TCE: traumatismo crânio-encefálico; N: número de pacientes; $p=$ significância 
Tabela 3 - Distribuição numérica e percentual dos grupos de pacientes em relação ao motivo da interrupção da intervenção fonoaudiológica $\left(p=0,009^{*}\right)$

\begin{tabular}{cccc}
\hline \multirow{2}{*}{ Patologia } & \multicolumn{3}{c}{ Alta } \\
\cline { 2 - 4 } & fonoaudiológica & hospitalar & óbito \\
\cline { 2 - 4 } & $\mathbf{N}(\%)$ & $\mathbf{N}(\%)$ & $\mathbf{N}(\%)$ \\
\hline AVE & $7(22,58)$ & $20(64,52)$ & $4(12,90)$ \\
TCE & $6(58,71)$ & $1(14,29)$ & $0(0)$ \\
Demência & $3(25)$ & $8(66,67)$ & $1(8,33)$ \\
\hline
\end{tabular}

Legenda: AVE: acidente vascular encefálico; TCE: traumatismo crânio-encefálico; N: número de pacientes

Tabela 4 - Média do número de atendimentos fonoaudiológicos, em dias, nos grupos de pacientes estudados $(p=0,401)$

\begin{tabular}{ccccc}
\hline \multirow{2}{*}{ Patologia } & \multicolumn{4}{c}{ Atendimentos } \\
\cline { 2 - 5 } & Média & Desvio-padrão & Mínimo & Máximo \\
\hline AVE & 15,84 & 10,57 & 3 & 47 \\
TCE & 16 & 9,02 & 4 & 32 \\
Demência & 12,25 & 8,68 & 3 & 27 \\
\hline
\end{tabular}

Legenda: AVE: acidente vascular encefálico; TCE: traumatismo crânio-encefálico

\section{DISCUSSÃO}

A aplicação da escala FOIS demonstrou que com a intervenção fonoaudiológica há um avançar significativo de níveis da escala por parte dos pacientes com disfagia orofaríngea e em âmbito hospitalar, que corresponde ao aumento da ingesta por via oral e ao maior número de consistências alimentares ingeridas. Essa evolução pode ser observada tanto em pacientes pós-AVE, como nos pós-TCE e naqueles com demência. Porém se observam particularidades em cada grupo. No grupo dos pacientes pós-TCE, todos iniciaram a intervenção fonoaudiológica fazendo uso de via alternativa de alimentação, e no final da intervenção a maioria alcançou o maior nível da escala, apresentando uma alimentação totalmente por via oral, sem restrições. No caso dos pacientes do grupo pós-AVE, a grande maioria destes também iniciou a intervenção com uso de vias alternativas, entretanto, diferenciando-se dos pacientes pós-TCE, a maioria dos pós-AVE permanecem no nível $V$ da escala, com alimentação com múltiplas consistências, mas que requerem um preparo especial, até o final da intervenção fonoaudiológica hospitalar. Já os pacientes com demência, que como nos demais grupos iniciam em sua maioria com o uso de vias alternativas, chegam ao final da intervenção em sua maioria no nível $\mathrm{V}$ da escala, todavia verificase maior percentual destes pacientes permane- cendo com o uso de via alternativa, até como um suplemento da alimentação por via oral.

Tais dados podem nos sugerir que nos três tipos de patologia os pacientes em sua maioria iniciam a intervenção fonoaudiológica com uso de vias alternativas, porém diferenciam-se no decorrer de tal intervenção, com o grupo pós-TCE, mesmo em número menor de sujeitos que os demais grupos, demonstrando menor tempo na reabilitação do processo de deglutição, pois alcançam níveis mais altos da escala durante a internação hospitalar, do que os pacientes pós-AVE que permanecem em níveis mais baixos da escala, necessitando de maiores preparos e cuidados durante a alimentação até o término do atendimento fonoaudiológico hospitalar. Nos pacientes com demência, os dados sugerem a necessidade freqüente do uso da via alternativa, em decorrência da confusão mental e progressão decorrente da patologia, o que dificulta uma boa aceitação alimentar, além de haver uma progressiva piora na funcionalidade da deglutição.

A presença do nível IV da escala FOIS, que representa a alimentação total por via oral, mas de uma única consistência, indicando o espessamento de líquidos até a consistência pastosa, ocorreu nos pacientes com demência e nos pós-AVE.

No estudo de Winstein et al ${ }^{12} \mathrm{em}$ pacientes pós-TCE, foi verificado que o aspecto cognitivo é um grande fator de interferência na reabilitação da deglutição nestes pacientes, com $84 \%$ destes recebendo alimentação totalmente por via oral. 
Crary et al ${ }^{13}$ em seu estudo verificaram que a escala FOIS foi significativa em correlacionar a disfagia com o grau de severidade do AVE, mas não em correlacionar a disfagia com o estado nutricional. De acordo com estudo da literatura, é alta a incidência de disfagia na fase aguda do AVE, independente da localização da lesão, indicando a necessidade da intervenção fonoaudiológica diante desses casos ${ }^{14}$. Shelp et al ${ }^{15}$ verifica a incidência de disfagia em pacientes pós-AVE em $76,5 \%$.

Neumann et al ${ }^{16}$ afirmam em seu estudo com pacientes pós-AVE ou pós-TCE que, independentemente do tempo de lesão neurológica, a atuação fonoaudiológica frente os distúrbios de deglutição sempre produz resultados, em maior ou em menor grau. Além disso, relatam que a terapia de deglutição reduz a incidência de BCP.

Estudos ${ }^{17-21}$ da literatura verificam que em pacientes com demência, conforme há o avançar da patologia, há aumento da dificuldade de deglutição e a perda do interesse em alimentar-se, com grandes chances de redução das complicações clínicas decorrentes da disfagia com a intervenção fonoaudiológica, sendo a pneumonia uma causa comum de morte em pacientes com demências, como a doença de Alzheimer e a demência frontotemporal.

Silva ${ }^{22}$ conclui que a eficácia da reabilitação nas disfagias orofaríngeas decorre de um programa terapêutico que selecione procedimentos capazes de produzir efeitos benéficos na dinâmica da deglutição, ocasionando alterações no aspecto geral do indivíduo. Marik et al ${ }^{23}$ e Bisch et al ${ }^{24}$ apontam o uso de estratégias compensatórias, como modificação de consistências e volumes alimentares e modo de oferta, assim como modificações de postura, para promover a alimentação e a eficiência da deglutição, e reduzir os riscos de aspiração. Cola et al ${ }^{25}$ indicam que o uso do sabor azedo e da temperatura fria auxilia de forma positiva a reabilitação da disfagia.

Furkim e Sacco ${ }^{26}$ em estudo com pacientes neurológicos e hospitalizados, usando a escala FOIS como indicador de evolução, encontraram que $73,5 \%$ dos 49 pacientes apresentaram avançar no nível da escala FOIS, indicando evolução com relação à funcionalidade da deglutição.

A redução significativa do percentual de broncopneumonias nos pacientes deste estudo, independente da patologia de base, pode demonstrar a eficácia da intervenção fonoaudiológica frente ao paciente com disfagia orofaríngea, além dos resultados obtidos com o uso de medicamentos e da intervenção da fisioterapia respiratória. A intervenção fonoaudiológica pode contribuir para a melhora clínica e pulmonar do paciente, com a redução dos episódios de penetração e/ou aspiração laringotraqueal, através da verificação da possibilidade de ingesta por via oral, indicando as consistências alimentares, utensílios e postura alimentar mais indicados em cada caso.

No estudo de Kasprisin et al ${ }^{27}$, os autores relatam que a eficácia da reabilitação dos distúrbios da deglutição é demonstrada pela diferença na ocorrência de BCP entre os pacientes reabilitados e aqueles que não passaram pelo processo de reabilitação, sugerindo que a reabilitação dos distúrbios da deglutição pode ser efetiva em pacientes que já apresentavam história prévia de BCP. Verificam que somente $6-15 \%$ dos pacientes reabilitados apresentam $\mathrm{BCP}$, em comparação a $75-100 \%$ dos pacientes que não passaram pela reabilitação. Neste mesmo estudo, encontra-se que a pneumonia aspirativa ocorre nos diversos níveis de severidade de disfagia, indicando que todos os pacientes disfágicos apresentam risco potencial para o desenvolvimento desta. Demonstram que a ocorrência de BCP aproximadamente dobra comparando-se os níveis leve e levemente moderado de comprometimento da deglutição, e aumenta em $70 \%$ comparando-se os níveis levemente moderado e moderado. Outros estudos $28-30$ também afirmam que há redução na ocorrência de BCP em pacientes que recebem reabilitação frente os distúrbios de deglutição. Power et al ${ }^{31}$ referem à importância da atuação à beira do leito para a redução da aspiração.

O motivo da interrupção da intervenção fonoaudiológica também se diferenciou nos três grupos, sendo que no grupo pós-TCE, o motivo mais freqüente foi a alta fonoaudiológica, demonstrando que os pacientes deste estudo alcançaram a funcionalidade e eficácia da deglutição ainda durante a internação hospitalar. Já os pacientes pós-AVE e os com demência apresentaram como mais freqüente motivo a alta hospitalar, indicando que ainda haviam objetivos fonoaudiológicos no trabalho de reabilitação da deglutição nestes pacientes. Os óbitos estiveram presentes também nestes dois últimos grupos. Vale dizer que a alta fonoaudiológica também ocorreu, mesmo que em menor percentual, nos pacientes pós-AVE, verificando que também há a reabilitação da funcionalidade da deglutição destes pacientes ainda em âmbito hospitalar. A alta fonoaudiológica também observada nos com demência, ocorreu possivelmente devido à indicação da melhor via e consistências alimentares, e a ausência de objetivos para o trabalho fonoaudiológicos nestes pacientes, devido à progressão da patologia.

Cabe dizer, que o último dia de atendimento fonoaudiológico pode coincidir ou não com a alta 
hospitalar do paciente, muitas vezes ainda havendo objetivos quanto à atuação fonoaudiológica frente ao caso.

Klor et al ${ }^{32}$ sugerem em seu estudo que os pacientes com disfagia podem ser beneficiados de forma significativa pela intervenção fonoaudiológica frente aos distúrbios da deglutição, promovendo o retorno à alimentação por via oral de forma mais rápida, contribuindo com os custos hospitalares, uma vez que o custo da alimentação por via oral representa $21-31 \%$ do custo da alimentação enteral. Segundo Smithard et al ${ }^{30}$, os pacientes com disfagia apresentam maior período de internação hospitalar (44,8 dias, comparados com 24,5 dias de pacientes não disfágicos). Estudo ${ }^{33}$ afirma que há evidências que a detecção precoce do disfagia em pacientes pós-AVE reduz não somente as complicações clínicas, como também o período de internação e os gastos hospitalares.
A média do número de atendimentos fonoaudiológicos foi muito semelhante em todos os grupos, porém, com os demais dados, pode-se sugerir que a maior parte dos pacientes pós-AVE e os com demência necessitariam de maior tempo de intervenção para atingir a eficácia do processo da deglutição.

\section{CONCLUSÃO}

Os pacientes hospitalizados com disfagia orofaríngea, nas três patologias estudadas, demonstraram avançar das consistências alimentares na ingesta oral, em diferentes níveis, e redução da ocorrência de broncopneumonias, após a intervenção fonoaudiológica com relação aos distúrbios da deglutição.

\begin{abstract}
Purpose: to investigate the development in oral intake and the incidence of bronchopneumonia $(\mathrm{BCP})$ in hospitalized patients with neurogenic oropharyngeal dysphagia, after speech and language therapy intervention. Methods: 50 adult patients, divided in three groups: I: 31 post stroke patients; II: seven brain injury patients ; III: 12 dementia patients. Data collected before and after the speech and language therapy intervention were: staff classification in Functional Oral Intake Scale (FOIS), incidence of BCP, number of therapies and reason for their interruption. Results: significant increase in the levels of FOIS scale and reduction in incidence of pneumonia in the three studied groups. In the post stroke and dementia groups the reason for therapy interruption was hospital discharge, and in the group of brain injury the reason was speech and language therapy discharge. Conclusion: the studied patients show increase in food consistency as for oral intake and reductions of BCP after speech and language therapy intervention related to swallowing disorders.
\end{abstract}

KEYWORDS: Deglutition Disorders; Speech Therapy; Stroke; Brain Injuries; Dementia

\section{REFERÊNCIAS}

1. Padovani AR, Moraes DP, Mangili LD, Andrade CRF. Protocolo fonoaudiológico de avaliação do risco para disfagia (PARD). Rev Soc Bras Fonoaudiol. 2007; 12(3):199-205.

2. Maciel JRV, Oliveira CJR, Tada CMP. Associação entre risco de disfagia e risco nutricional em idosos internados em hospital universitário de Brasília. Rev Nutr. 2008;21(4):411-21.

3. Falsetti $P$, Acciai $C$, Palilla $R$, Bosi M, Carpinteri $F$, Zingarelli $A$, et al. Oropharyngeal dysphagia after stroke: incidence, diagnosis, and clinical predictors in patients admitted to a neurorehabilitation unit. J Stroke Cerebrovasc Dis. 2009;18(5):329-35.
4. Rocamora JAI, Sánchez-Duque MJ, Galindo PBV, López EB, Palacín AF, González CA, et al. A prevalence study of dysphagia and intervention with dietary counselling in nursing home from Seville. Nutr Hosp. 2009;24(4):498-503.

5. Silva ACV, Fabio SRC, Dantas RO. A Scintigraphic study of oral, pharyngeal, and esophageal transit in patients with stroke. Dysphagia 2008;23(2):165-171.

6. Teramoto S. Novel preventive and therapuetic strategy for post-stroke pneumonia. Expert Rev Neurother. 2009;9(8):1187-200.

7. Broadley S, Cheek, A, Salonikis S, Whitham E, Chong V, Cardone D, et al. Predicting prolonged dysphagia in acute stroke: the royal adelaide 
prognostic index for dysphagic stroke (RAPIDS). Dysphagia 2006;20(4):303-10.

8. Cook IJ. Oropharyngeal dysphagia. Gastroenterol Clin North Am 2009;38(3):411-31

9. DePippo KL, Holas MA, Reding Mj, Mandel FS, Lesser Ml. Dysphagia therapy following stroke: a controlled trial. Neurol. 1994; 44(9):1655-60.

10. Angelis EC, Fúria CLB. Tratamento fonoaudiológico em hospital oncológico: disfagia em câncer de cabeça e pescoço. In: Hernandez AM, Marchesan I. Atuação fonoaudiológica no ambiente hospitalar. Rio de Janeiro: Revinter; 2001. p.81.

11. Crary MA, Mann GDC, Groher ME. Initial psychometric assessment of a functional oral intake scale for dysphagia in stroke patients. Arch Phys Med Rehabil. 2005; 86:1516-20.

12. Winsteins Cl. Neurogenic dysphagia: frequency, progression, and outcome in adults following head injury. Phys Ther. 1983; 63:1992-6.

13. Crary MA, Carnaby-Mann GD, Miller L, Antonios $\mathrm{N}$, Silliman S. Dysphagia and nutritional status at the time of hospital admission for ischemic stroke. J Stroke Cerebrovascular Dis. 2006; 15(4):164-71. 14. Barros AFF, Fábio SRC, Furkim AM. Correlação entre os achados clínicos da deglutição e os achados da tomografia computadorizada de crânio em pacientes com acidente vascular cerebral isquêmico na fase aguda da doença. Arq Neuropsiquiatr. 2006; 64(4):1009-14.

15. Schelp AO, Cola PC, Gatto AR, Silva RG, Carvalho LR. Incidência de disfagia orofaríngea após acidente vascular encefálico em hospital público de referência. Arq Neuro-Psiquiatr. 2004; 62(2-B):503-6.

16. Neumann S, Bartolome G, Buchholz D, Prosiegel M. Swallowing therapy of neurologic patients: correlation of outcome with pretreatment variables and therapeutic methods. Dysphagia. 1995; 10:1-5. 17. Easterling CS, Robbins E. Dementia and dysphagia. Geriatr Nurs. 2008;29(4):275-85.

18. Shinagawa S, Adachi $\mathrm{H}$, Toyota $\mathrm{Y}$, Mori $\mathrm{T}$, Matsumoto I, Fukuhara R. Characteristics of eating and swallowing problems in patients who have dementia with Lewy bodies. Int Psychogeriatr 2009;21(3):520-25.

DOI: 10.1590/S1516-18462010005000090

RECEBIDO EM: 24/06/2009

ACEITO EM: 18/12/2009

Endereço para correspondência:

Carolina Castelli Silvério

Rua Botucatu, 261 ap. 24

São Paulo - SP

CEP: 04023-061

E-mail: carol_silverio@ hotmail.com
19. Suh MK, Kim HH, Na DL. Dysphagia in patients with dementia: Alzheimer versus vascular. Alzheimer Dis Assoc Disord 2009;23:178-84.

20. Sharp HM, Shega JW. Feeding tube placement in patients with advanced dementia: the beliefs and practice patterns of speech-language pathologists. Am J Speech Lang Pathol. 2009;18(3):222-30.

21. Langmore SE, Olney RK, Lomen-Hoerth C, Miller B. Dysphagia in patients with frontotemporal lobar dementia. Arch Neurol. 2007; 64:58-62.

22. Silva RG. A eficácia da reabilitação em disfagia orofaríngea. Pró-Fono. 2007; 19(1):123-31.

23. Marik PE, Kaplan D. Aspiration pneumonia and dysphagia in the elderly. Chest. 2003; 124:328-36.

24. Bisch EM, Logemann J, Rademaker AW, Kahrilas PJ, Lazarus CL. Pharyngeal effects of bolus volume, viscosity, and temperature in patients with dysphagia resulting from neurologic impairment and in normal subjects. J Speech Hear Res. 1994; 37(5):1041-59.

25. Cola PC, Gatto AR, Silva RG, Schelp AO, Henry MACA. Reabilitação em disfagia orofaríngea neurogênica: sabor azedo e temperatura fria. Rev CEFAC. 2008; 10(2):200-5.

26. Furkim AM, Sacco ABF. Eficácia da fonoterapia em disfagia neurogênica usando a escala funcional de ingestão por via oral (FOIS) como marcador. Rev CEFAC. 2008; 10(4):503-12.

27. Kasprisin AT, Clumek H, Nino-Mureia M. The efficacy of rehabilitative management of dysphagia. Dysphagia. 1989; 4:48-52.

28. Langdon PC, Le AH, Binns CW. Dysphagia in acute ischaemic stroke, recovery and relationship to stroke subtype. J Clin Neurosci. 2007; 14:630-4.

29. Hinchey JA, Shephard T, Furie K, Smith D, Wang D, Tonn S. Formal dysphagia screening protocols prevent pneumonia. Stroke. 2005; 36:1972-6.

30. Walter U, Knoblich R, Steinhagen V, Donat M, Benecke R, Kloth A. Predictor of pneumonia in acute stroke patients admitted to a neurological intensive care unit. J Neurol. 2007; 254:1323-9.

31. Power ML, Fraser CH, Hobson A, Singh S, Tyrrell $P$, Nicholson DA, et al. Evaluating oral stimulation as a treatment for dysphagia after stroke. Dysphagia 2006;21(1):49-55.

32. Klor BM, Milianti FJ. Rehabilitation of neurogenic dysphagia with percutaneos endoscopic gastrostomy. Dysphagia. 1999; 14:162-4.

33. Martino R, Pron G Diamant NE. Screening for oropharyngeal dysphagia in stroke: insufficient evidence for guidelines. Dysphagia. 2000; (1):19-30. 